\title{
Erratum: Autonomously Probing Viscoelasticity in Disordered Suspensions [Phys. Rev. Lett. 125, 258002 (2020)]
}

Clara Abaurrea-Velasco, Celia Lozano, Clemens Bechinger, and Joost de Graaf®

(A) (Received 17 February 2021; published 15 March 2021)

DOI: 10.1103/PhysRevLett.126.119902

On page 1, we claim that "there is presently no microscopic understanding of what underlies the rotational diffusion enhancement in the glassy system." This is accurate when it comes to glasses, but does not do justice to the work of Ref. [1], which has shown enhancement of this kind in confined layers of monodisperse spheres using a squirmer model.

[1] J. L. Aragones, S. Yazdi, and A. Alexander-Katz, Phys. Rev. Fluids 3, 083301 (2018). 